\title{
Droplet formation in microfluidic T-junction generators operating in the transitional regime. I. Experimental observations
}

\author{
Tomasz Glawdel, Caglar Elbuken, and Carolyn L. Ren* \\ Department of Mechanical and Mechatronics Engineering, University of Waterloo, Waterloo, Ontario, Canada N2L3G1
}

(Received 12 October 2011; published 26 January 2012)

\begin{abstract}
This is the first part of a two-part study on the generation of droplets at a microfluidic T-junction operating in the transition regime where confinement of the droplet creates a large squeezing pressure that influences droplet formation. In this regime, the operation of the T-junction depends on the geometry of the intersection (height-to-width ratio, inlet width ratio), capillary number, flow ratio, and viscosity ratio of the two phases. Here in paper I we presented our experimental observations through the analysis of high-speed videos of the droplet formation process. Various parameters are tracked during the formation cycle such as the shape of the droplet (penetration depth and neck), interdroplet spacing, production rate, and flow of both phases across several T-junction designs and flow conditions. Generally, the formation process is defined by a two-stage model consisting of an initial filling stage followed by a necking stage. However, video evidence suggests the inclusion of a third stage, which we term the lag stage, at the beginning of the formation process that accounts for the retraction of the interface back into the injection channel after detachment. Based on the observations made in this paper, a model is developed to describe the formation process in paper II, which can be used to understand the design and operation of T-junction generators in the transition regime.
\end{abstract}

DOI: 10.1103/PhysRevE.85.016322

PACS number(s): 47.61.Jd, 47.55.db

\section{INTRODUCTION}

Droplet-based microfluidic devices have garnered a great deal of attention recently due to their enormous potential in large-scale combinatorial studies that require high throughput and the foremost control over droplet size [1-5]. One of the most common droplet generator designs used in these devices is the T-junction configuration (see Fig. 1) [5]. In this design, the dispersed phase microchannel, usually containing an aqueous solution, perpendicularly intersects the main channel containing the continuous phase (oil). Forces created by the cross-flowing continuous phase periodically break off droplets as the dispersed phase is injected into the main channel. The operational regime is primarily characterized by the capillary number $\mathrm{Ca}=\mu U / \gamma$, which quantifies the competition between the viscous shear and interfacial forces. Three operational regimes have been identified where breakup is dominated by either confinement of the emerging droplet within the microchannel, known as the squeezing regime (low $\mathrm{Ca}$ ), or the balance of shear and surface tension forces, known as the dripping regime (high $\mathrm{Ca}$ ). In between the two regimes exists the transition regime, where both forces are important [1,5-9].

Droplet formation in the T-junction is generally regarded as consisting of two steps, as illustrated in Fig. 1. (i) A cycle begins with the filling period, where the dispersed phase is injected from the side channel into the main flow. The droplet grows until it reaches a maximum penetration depth $b$, which is determined by the forces acting on the emerging interface [1]. During this time, the continuous phase bypasses the droplet and fills the space between the previously formed droplet, thus increasing the distance between them. (ii) The obstruction causes pressure to build upstream, which begins to squeeze the neck, causing it to collapse. At the same time the dispersed

\footnotetext{
*c3ren@uwaterloo.ca
}

phase is still being pumped into the droplet and the size increases [7,10,11]. Eventually the neck reaches a critical size where the rate of collapse accelerates dramatically and the droplet detaches [12]. The newly formed droplet is pushed downstream as the interface recoils and the process repeats. The final size of the droplet is the sum of the volume at the end of the filling stage and the additional volume pumped in before the neck collapses. The scaling law that captures the physics of this process has the general form $V_{d}{ }^{*}=\alpha+\beta \varphi$, where $V_{d}{ }^{*}=$ $V_{d} / w_{c}{ }^{2} h$ is the dimensionless volume, $\varphi$ is the flow rate ratio $Q_{d} / \mathrm{Q}_{c}$, and the variables $\alpha$ and $\beta$ represent the dimensionless initial filling volume and necking periods $[7,13]$.

The parameters governing droplet formation consist of the geometry of the T-junction $w_{c}, w_{d}$, and $h$ and the flow conditions $Q_{c}, Q_{d}, \gamma, \mu_{c}, \mu_{d}$, and $\rho$ (refer to Fig. 1 for definitions). Based on the Buckingham $\Pi$ theorem, the dimensionless volume can be described by six independent dimensionless groups $[6,14]$

$$
\begin{aligned}
\varphi & =\frac{Q_{d}}{Q_{c}}, \quad \eta=\frac{\mu_{d}}{\mu_{c}}, \quad \Lambda^{*}=\frac{w_{d}}{w_{c}}, \quad h^{*}=\frac{h}{w_{c}}, \\
\mathrm{Ca} & =\frac{\mu_{c} Q_{c}}{\gamma w_{c} h}, \quad \operatorname{Re}=\frac{\rho Q_{c}}{h \mu_{c}},
\end{aligned}
$$

where $\varphi$ is the flow ratio, $\eta$ is the viscosity contrast, $\Lambda^{*}$ is the intersection ratio, $h^{*}$ is the height-to-width ratio, $\mathrm{Ca}$ is the capillary number, and Re is the Reynolds number. For typical microchannel flows, the Reynolds number is not important $(\operatorname{Re} \ll 1)$ and the volume is therefore a function of the five remaining groups: $V_{d}^{*}=f\left(\phi, \eta, \Lambda^{*}, h^{*}, \mathrm{Ca}\right)$. Assuming that the scaling law is applicable, $\alpha$ and $\beta$ are also dependent on these dimensionless factors.

A recent review by Steegmans et al. compared the suitability of several models by statistically evaluating their predictive ability [15]. Generally, the models performed well with respect to their own data set, but poorly against external data sets. The major issue is that many of the models treat $\alpha$ and $\beta$ as fitting 


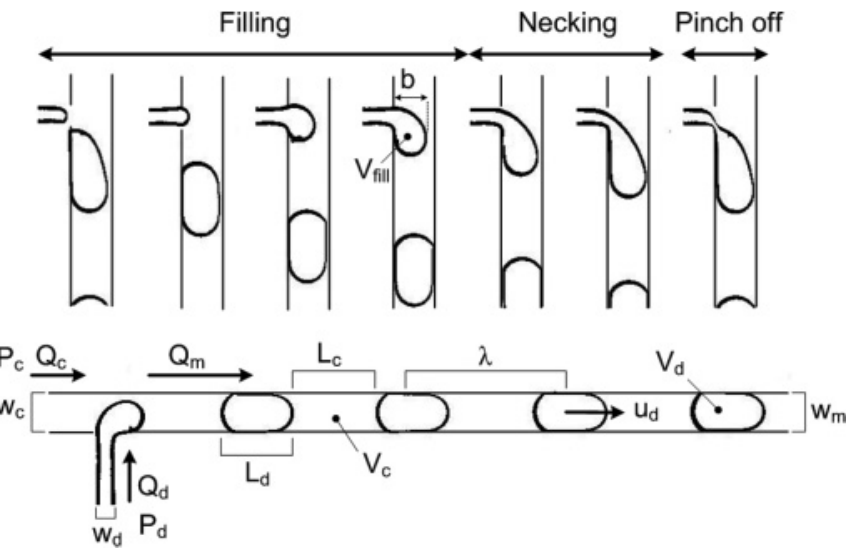

FIG. 1. Droplet formation cycle in the T-junction generator consisting of three stages: the filling period, squeezing or necking period, and pinchoff. Images are actual traces of the interface with intersection geometry $\left(w_{d}: w_{c}\right) 1: 2$ and channel height $50 \mu \mathrm{m}$, under flow conditions of $\mathrm{Ca}=0.0087$ and $\varphi=0.475$. All images are equidistant in time. Below, the global overview of the T-junction generator is shown with the respective geometric and flow parameters identified. Definitions include the flow rates $Q$ and widths $w$ of the continuous, dispersed, and main channels and the height $h$, which is uniform throughout the network. Droplets are formed with a length $L_{d}$ and volume $V_{d}$, at a frequency $f$ and spacing $\lambda$, and are transported along the channel at a velocity $u_{d}$. The space between the droplets occupied by the continuous fluid is $L_{c}$ and has a volume $V_{c}$. Several dimensionless terms define the operation of the generator, including the width ratio $\Lambda^{*}=w_{d} / w_{c}$, height-to-width ratio $h^{*}=h / w_{c}$, flow ratio $\varphi=Q_{d} / Q_{c}$, and viscosity ratio $\eta=\mu_{d} / \mu_{c}$.

parameters in the development of empirical correlations for the specific T-junction design under study $[8,11,16-20]$. These correlations take the general form of $V_{d}{ }^{*}=\alpha^{a} \mathrm{Ca}^{b}+\beta^{c} \mathrm{Ca}^{d} \varphi^{e}$, where $a-e$ are fitting parameters that attempt to capture the various parametric influences observed in experiments. The disparity between various correlations suggests that they are limited to the specific geometries and conditions upon which they were developed.

Several works have also developed physical models that describe the phenomena governing droplet formation. Models in the dripping regime use a detailed force balance to derive the final droplet size [21-23]. In the transition regime, the most notable work is by Christopher et al., who developed a force balance model to determine the filling volume and incorporated geometry into the two formation parameters [1]. When compared with experimental results, the model captures many of the salient features that are often observed, including the $\mathrm{Ca}$ and geometric dependence on the size of the droplets. Others have applied the model to numerical [14,24] and experimental $[11,25,26]$ data with reasonable agreement, yet discrepancies still arise, suggesting that improvements can be made.

Recently, van Steijn et al. developed a model for the two parameters in the squeezing regime that combines a detailed geometric representation of the droplet with the application of physical principles to describe the formation process [13]. The model is composed of three parts: (i) a three-dimensional description of the droplet from the initial filling stage to the necking stage, (ii) the application of the continuity equation to derive the evolution of the neck during the squeezing period, and (iii) the incorporation of a pinchoff mechanism to define the end of the necking stage. The result is a series of nontrivial equations that describe the two parameters $\alpha$ and $\beta$ based entirely on the geometry of the channel $\left(\Lambda^{*}=w_{d} / w_{c}\right.$ and $\left.h^{*}=h / w_{c}\right)$. Although remarkable in its predictive ability in the squeezing regime, the model is not applicable in the transitional regime, where droplet formation also depends on flow conditions. Thus a comprehensive model that can predict droplet generation into the squeezing-to-transition regime is still required [25].

Our goal is to describe all aspects of generator performance including droplet size, interdroplet spacing, and frequency of formation within the transition regime. This paper is the first of a two-part series that describes the development of a transition model. In this paper we first analyze high-speed videos of droplet formation in detail to understand the underlying processes involved. For this purpose, we track various parameters during the formation cycle such as the shape of the droplet, interdroplet spacing, and flow of both phases. We analyze the dependence of these parameters on both geometry and flow conditions and develop scaling arguments for the operational parameters. Our observations suggest the inclusion of a third stage at the beginning of droplet formation that we term the lag stage. In paper II we develop a model that describes the overall operation of T-junction generators and validate it against experimental data [26].

\section{METHODS AND MATERIALS}

Our experimental study was designed to span typical conditions in T-junction generators by varying the capillary number, width ratio, channel height, and viscosity contrast. Each parameter is detailed below and experimental conditions are listed in Table I.

(i) Continuous phase. For all experiments the continuous phase was a low-viscosity silicone oil (DC200, Sigma Aldrich). Silicone oil swells poly(dimethyl)siloxane (PDMS) slightly. which changes the dimensions of the channels [27]. For this reason, the dimensions of the channels $(w, h)$ were measured carefully before each experiment [28].

(ii) Dispersed phase. The dispersed phase was a set of glycerol-water mixtures of 10, 30, 50, and 70 wt.\%. Adding glycerol to water increases the viscosity of the dispersed phase without changing the interfacial tension appreciably. With these combinations the viscosity ratio between the dispersed and continuous phase $\eta=0.12,0.26,0.6$, and 1.7 .

TABLE I. List of conditions for each experimental case study.

\begin{tabular}{llll}
\hline \hline Expt. No. & $\Lambda^{*}$ & $h^{*}$ & $\eta$ \\
\hline $1-3$ & 0.3 & 0.3 & $0.12,0.26,0.6$ \\
$4-6$ & 1 & 0.3 & $0.12,0.26,0.6$ \\
$5-9$ & 0.3 & 0.5 & $0.12,0.26,0.6$ \\
$10-12$ & 0.5 & 0.5 & $0.12,0.26,0.6$ \\
$13-16$ & 1 & 0.6 & $0.12,0.26,0.6,1.7$ \\
$17-20$ & 0.5 & 0.6 & $0.12,0.26,0.6,1.7$ \\
$21-22$ & 1 & 0.6 & $0.12,0.26$ \\
\hline \hline
\end{tabular}


TABLE II. Properties including viscosity and interfacial tension for each dispersed-continuous phase combination. Measurements are made at room temperature $22 \pm 1^{\circ} \mathrm{C}$.

\begin{tabular}{cccccc}
\hline \hline \multicolumn{2}{c}{ Continuous phase } & & \multicolumn{2}{c}{ Dispersed phase } & \\
\cline { 1 - 3 } & $\begin{array}{c}\text { Viscosity } \\
(\mathrm{mPa} \mathrm{s})\end{array}$ & & Glycerol & $\begin{array}{c}\text { Viscosity } \\
(\mathrm{mPa} \mathrm{s})\end{array}$ & $\begin{array}{c}\text { Interfacial tension } \\
(\mathrm{mN} / \mathrm{m})\end{array}$ \\
\hline silicone oil & 10.2 & $10 \%$ & 1.21 & $37.2 \pm 0.01$ \\
& & $30 \%$ & 2.61 & $36.3 \pm 0.02$ \\
& & $50 \%$ & 6.05 & $35.4 \pm 0.01$ \\
& & $70 \%$ & 17.1 & $34.1 \pm 0.01$ \\
\hline \hline
\end{tabular}

(iii) Width ratio and height-to-width ratio. The crosssectional shape of the microchannels throughout the network is rectangular with a uniform depth. The continuous phase channel and main channel width is kept constant at $100 \mu \mathrm{m}$, while the width of the dispersed phase inlet is set at 33,50 , and $100 \mu \mathrm{m}$, corresponding to width ratios of $\Lambda^{*}=0.33,0.5$, and 1. Three nominal channel heights were chosen: 30, 50, and $60 \mu \mathrm{m}$. This corresponds to aspect ratios $h^{*}=0.3,0.5$, and 0.6 .

The viscosity of each fluid was measured using a programmable rheometer (LVDV-III Ultra CPE, Brookfiled Instruments) with a cone-plate spindle (CPE-40) rated for a measurement range of 0.1-3070 $\mathrm{mPa}$ s. Measurements were made over a range of applied shear stresses to verify that each fluid exhibits Newtonian behavior. Interfacial tension was measured using the Wilhemy Plate method (Data Physics DCAT 11). All measurements were done in triplicate and are summarized in Table II.

\section{A. Chip fabrication}

Poly(dimethyl)siloxane chips are fabricated using standard soft-lithography methods from SU-8/silicon masters. Poly(dimethyl)siloxane molds were bonded to a glass slide coated with PDMS to create homogeneous microchannels. Poly(dimethyl)siloxane coated slides are fabricated by spin coating 10:1 PDMS at $3000 \mathrm{rpm}$ for $60 \mathrm{~s}$ followed by baking for 5 min at $95^{\circ} \mathrm{C}$. For bonding, the two substrates are exposed to oxygen plasma (PDC-001, Harrick Plasma) under conditions of $29.6 \mathrm{~W}$ at $600 \mathrm{mTorr}$ for $15 \mathrm{~s}$. The plasma treatment renders the PDMS hydrophilic; in order to reverse the process and make the channels wet to silicone oil the chip was heated at $180^{\circ} \mathrm{C}$ for $12 \mathrm{~h}$. Before starting an experiment silicone oil was pumped for $1 \mathrm{~h}$ to prime the microchannels.

\section{B. Experimental procedure}

Each chip was mounted onto an inverted epifluorescence microscope system (Eclipse Ti, Nikon) connected to a highspeed camera (Phantom v210, Vision Research). At full resolution $(1280 \times 800)$ the camera can take images at 2190 fps, with a 12-bit digital image quality. Fluids are controlled using a high-precision microfluidic pressure control system that operates up to 1 bar (MFSC $8 \mathrm{C}$, Fluigent). The capillary tubing connected to the chip has a large inside diameter (500 $\mu \mathrm{m}$ ) compared to the microchannels to minimize any pressure loss before the chip inlets. A custom coded acquisition program was used to automate the experiment (Labview v8.6, National Insturments). The dispersed phase flow rate is measured from the high-speed video analysis by assuming that mass is conserved by the droplets $Q_{d}=V_{d} f$, where $f$ is the frequency of formation. Droplet volumes are calculated using the projected area and perimeter subtraction method [13]. The continuous phase flow is measured directly using an in-line microflow sensor (SLG 1430-4870, Sensirion) that was recalibrated for silicone oil.

For each experiment different pressure combinations were applied to span a range of $\mathrm{Ca}$ and flow ratios. Videos and flow rates were recorded after $3 \mathrm{~min}$ of applying the pressures to make certain the system was at steady state. Afterward, videos were analyzed using a custom program (MATLAB, Mathworks) to extract operational parameters including penetration depth, neck pinchoff thickness, droplet size, speed, spacing, and frequency of production. Additional details on the video analysis are available in Ref. [28].

\section{EXPERIMENTAL OBSERVATIONS}

Figure 2 presents the drop formation process for four different T-junction geometries (cases A-D) for the same fluid combinations $(\eta=0.12)$. Cases $\mathrm{A}$ and $\mathrm{B}$ demonstrate the influence of intersection geometry on droplet production. In these two cases the flow conditions $\left(Q_{d}, Q_{c}\right.$, and Ca) are similar, but the width of the dispersed phase and the height are different. Case A produces smaller droplets at a higher frequency compared to case B. It is well known that the width ratio $\Lambda^{*}$ is a key factor that defines the operational performance of the T-junction generator. Designs with narrower inlet widths (case A) result in smaller droplets produced at higher rates with shorter interdroplet spacings. Alternatively, by changing the flow rates of the two phases, the droplet size, spacing, and rate of production can be tuned as shown in cases $C$ and D.

To provide further insight into the drop formation process we track the evolution of the droplet volume $V_{d}$ and shape $\left(b, 2 r_{n}\right)$ as well as the continuous phase volume $V_{c}$, which we decompose into the neck volume $V_{\text {neck }}$ and the volume between the new and old droplet $V_{\text {bypass }}$ for cases $\mathrm{A}$ and $\mathrm{B}$ as shown in Fig. 3. We define the distance that the interface penetrates into the main channel stream by the variable $b$ and the thickness of the neck $2 r_{n}$ as the distance at a $45^{\circ}$ angle from the inner corner of the junction to the back half of the droplet. By tracking changes in these volumes, the dispersed flow rate $Q_{d}$ and the fraction of the continuous phase flow $Q_{c}$ collapsing the neck $Q_{\text {neck }}$ or bypassing the droplet $Q_{\text {bypass }}$ can be measured. In the following discussion, we denote dimensionless values by an asterisk, where lengths are normalized by $w_{c}$, volumes by $w_{c}^{2} h$, and time by the period of formation $\tau_{\text {drop }}$.

Our analysis of the video evidence suggests that the formation process can be divided into three distinct periods denoted as lag, filling, and necking stages. The lag stage adds another element to the conventional two-stage model presented by other researchers. We summarize our observations and analysis of each stage below and suggest that the reader refer to Figs. 2 and 3 regularly in the following discussion.

\section{A. Lag stage}

Immediately after detachment, the interface recedes a small distance $L_{\text {lag }}$ back into the dispersed phase inlet before it 


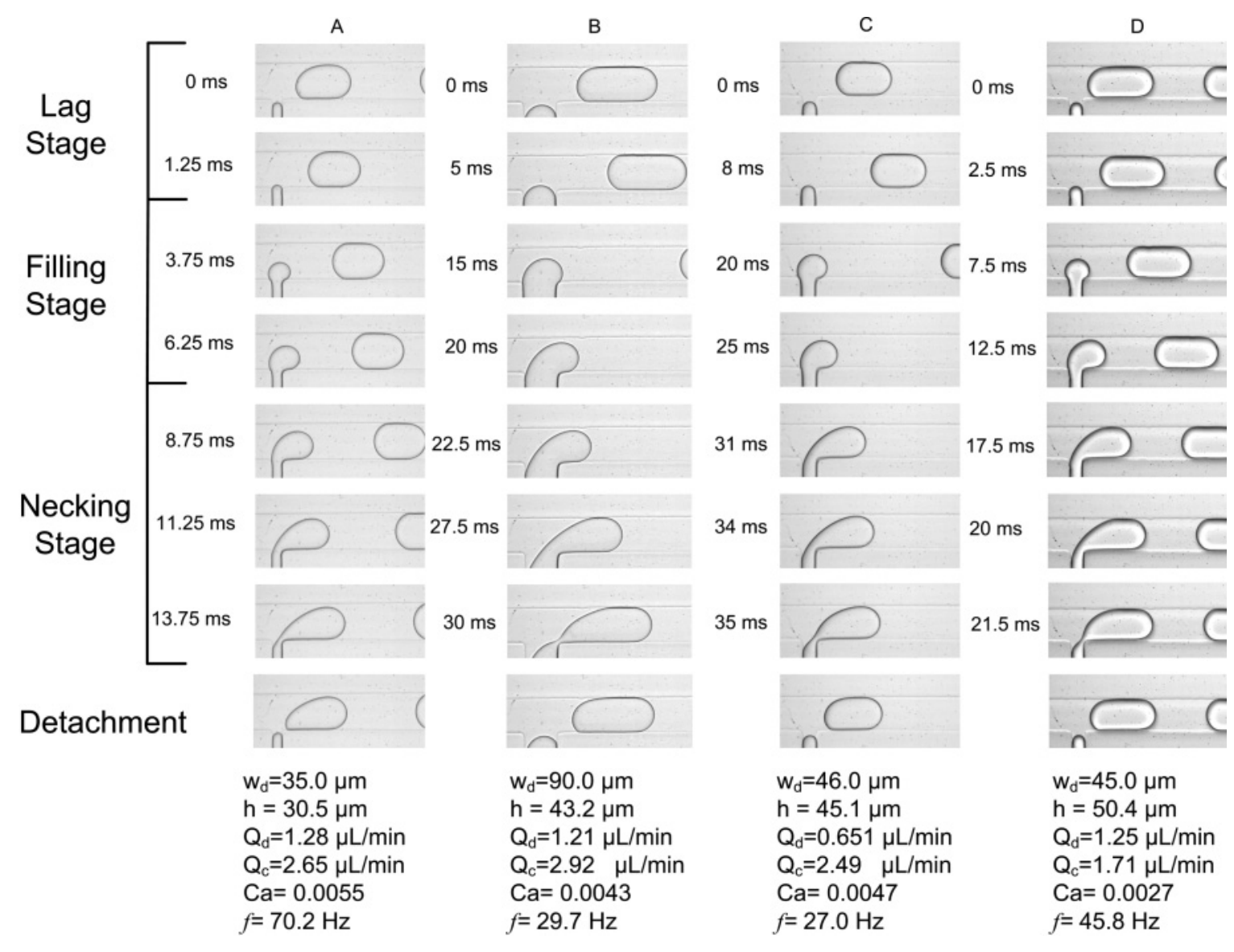

FIG. 2. Images of the drop formation process for four experiments broken down into three identifiable stages: lag, filling, and necking. Silicone oil is the continuous phase and $10 \mathrm{wt} . \%$ glycerol-water mixture is the dispersed phase; other experimental data are listed beside the images.

moves forward again and reaches the entrance of the main channel. The lag stage is typically very short, representing $2-10 \%$ of the total formation time and a small portion of the total droplet volume as well. All of the continuous phase bypasses the interface and fills the space behind the previously generated droplet increasing $V_{\text {bypass. }}$. The duration of the lag stage correlates well with the estimate from the flow rate $\Delta t_{\text {lag }}=L_{\text {lag }} /\left(Q_{d} / w_{d} h\right)$. Therefore, the droplet volume is expected to scale as $V_{\text {drop }} \sim L_{\text {lag }}$ and $V_{\text {bypass }} \sim \Delta t_{\text {lag }} Q_{c}$.

\section{B. Filling stage}

The interface now penetrates into the main channel and progresses towards the far wall. As the gap closes, hydrodynamic forces exerted on the droplet cause it to deform and it takes on a characteristic shape with a small radius at the front and a large radius at the back.

In the early filling stage $2 r_{n}$ increase sharply, but soon the rate of growth decreases, reaching a plateau, and then decreases at a linear rate in the necking stage. This transition between the plateau and the subsequent decrease in the neck thickness marks the end of the filling stage; it also marks a point where capillary and drag forces on the droplet balance. The characteristic shape of the droplet at the end of the filling stage is given by $b_{\text {fill }}$ and the droplet volume is $V_{\text {fill }}$.
For the examples, the penetration depth equals $b_{\text {fill }}^{*}=0.64$ for case $\mathrm{A}$ and $b_{\text {fill }}^{*}=0.83$ for case $\mathrm{B}$ even though the flow conditions are similar. The smaller $b_{\text {fill }}^{*}$ for case A results in a comparatively shorter filling stage and smaller droplet fill volumes (case A, $V_{\text {fill }}^{*} \sim 40 \% V_{\text {drop }}^{*}$; case B, $V_{\text {fill }}^{*} \sim 52 \% V_{\text {drop }}^{*}$ ). Therefore, one expects the duration of the filling stage to last $\Delta t_{\text {lag }} \sim V_{\text {fill }} / Q_{d}$.

During this same period of time the continuous phase keeps bypassing the droplet as $V_{\text {bypass }}$ increases linearly with time at a rate $d V / d t \sim Q_{c}$ while $V_{\text {neck }}$ remains relatively constant. Thus the bypass volume increases by $V_{\text {bypass }} \sim \Delta t_{\text {lag }} Q_{c}=$ $V_{\text {fill }} Q_{c} / Q_{d}$. In fact, during the entire formation process $V_{d}$ and $V_{c}$ increases at a linear rate, confirming that $Q_{d}$ and $Q_{c}$ are constant even though our experimental setup uses pressure-driven flow rather than syringe-pump-driven flow.

\section{Necking stage}

The transition into the necking stage results in a redistribution of $Q_{c}$ as a large fraction of the flow is directed to collapsing the neck. The amount collapsing the neck correlates with the degree of blockage created by the emerging interface: $b_{\text {fill }}^{*}=0.64$ and $d V_{\text {neck }} / d t \sim 0.55 Q_{c}$ for case $\mathrm{A}$ and $b_{\text {fill }}^{*}=0.82$ and $d V_{\text {neck }} / d t \sim 0.80 Q_{c}$ for case $\mathrm{B}$. The remaining fraction of the continuous phase keeps bypassing the droplet, thus increasing the forward oil volume. 


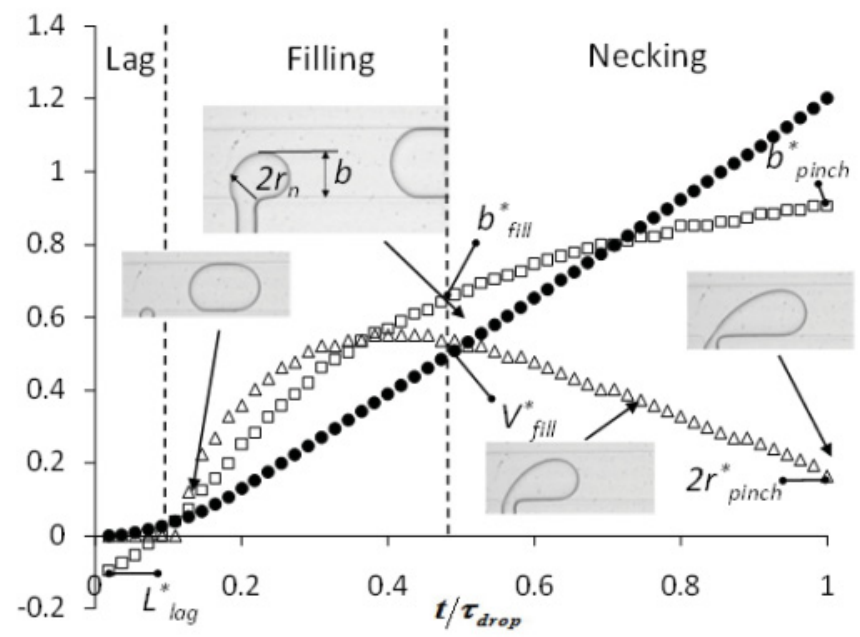

(a)

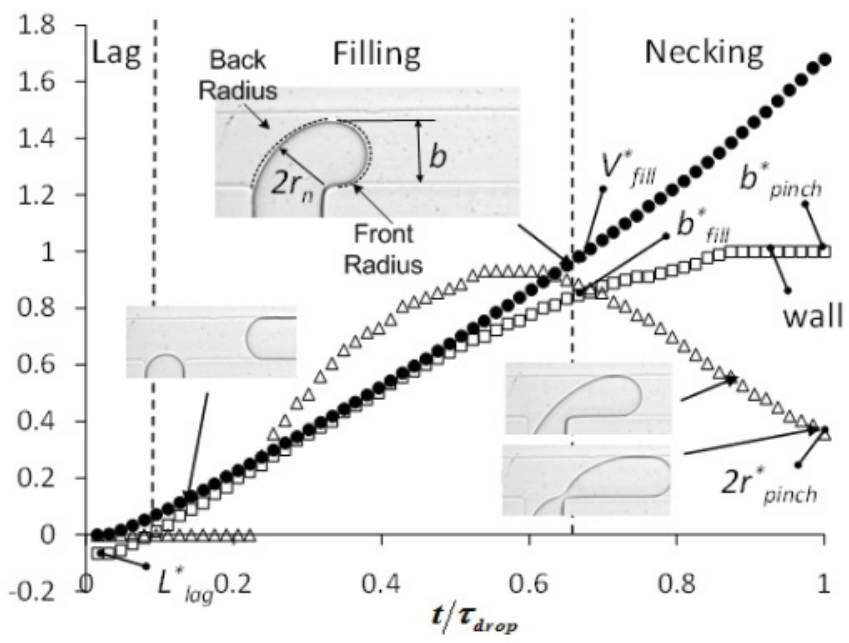

(c)

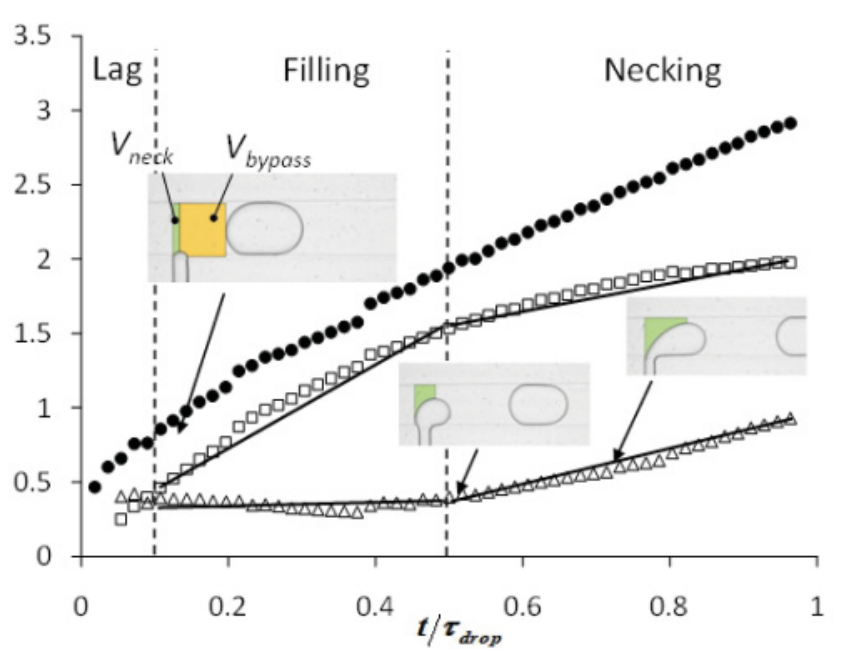

(b)

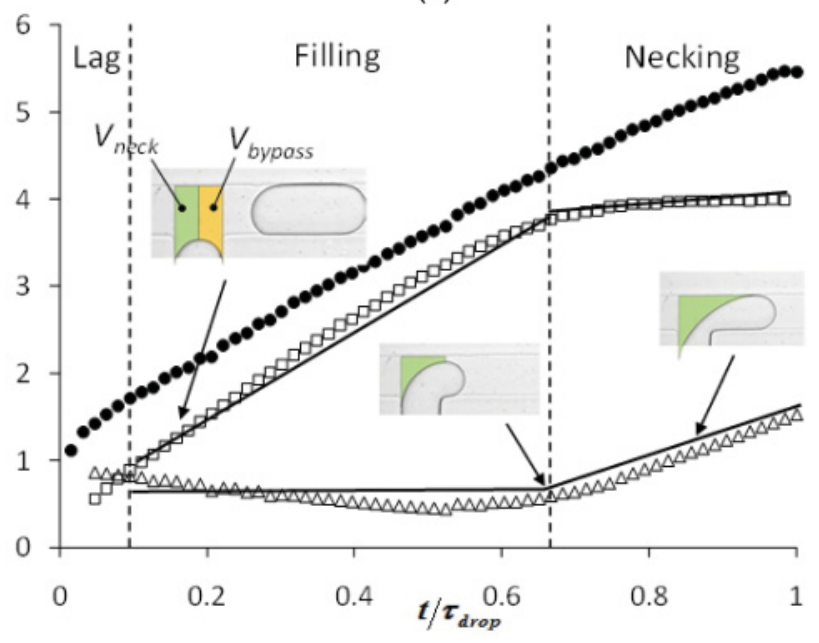

(d)

FIG. 3. (Color online) Evolution of the droplet shape and volume with time for cases A and B: changes in (a) and (c) $V_{\text {drop }}^{*}(\bullet), 2 r_{n}^{*}(\triangle)$, and $b^{*}(\square)$; (b) and (d) $V_{c}^{*}(\bullet), V_{\text {neck }}^{*}(\Delta)$, and $V_{\text {bypass }}^{*}(\square)$. Formation is characterized by the lag stage, filling stage, and necking stage as indicated by the dashed lines. Solid lines provide linear fits to the change in volume for the specified regions.

During necking the radius of the back half of the droplet increases as it is pushed toward the inner corner of the Tjunction. This continues until the neck thickness reaches a critical size $2 r_{\text {pinch }}$, where the droplet snaps off suddenly. This occurs at $2 r_{\text {pinch }}^{*}=0.16$ for case $\mathrm{A}$ and at $2 r_{\text {pinch }}^{*}=0.28$ for case B. Both these values are less than the estimate put forth by van Steijn et al. [12]: $2 r_{\text {pinch }}^{*}=0.23$ for case A and $2 r_{\text {pinch }}^{*}=$ 0.31 for case B. The smaller $2 r_{\text {pinch }}^{*}$ and lower $d V_{\text {neck }} / d t$ result in a comparatively longer necking stage for case A compared to case $\mathrm{B}$.

At the same time the droplet continues to grow in size. Growth is directed more toward the downstream direction as the length of the droplet increases. Nevertheless, the interface still continues to progress toward the far wall as $b$ increases due to a rebalancing of forces on the droplet. For case B the interface actually reaches the far channel wall before pinchoff. We designate the interface penetration at pinchoff as $b_{\text {pinch }}$ and later identify its importance in determining pinchoff.

The duration of the necking stage scales as $\Delta t_{\text {neck }} \sim$ $\Delta V_{\text {neck }} / Q_{c}$ and the droplet volume increases as $V_{\text {drop }} \sim$
$\Delta t_{\text {neck }} Q_{d} \sim \Delta V_{\text {neck }} Q_{d} / Q_{c}$, demonstrating the dependence of droplet size on flow ratio in the necking stage. Necking ends once the droplet detaches and the formation process repeats, beginning with the lag stage.

Our analysis of the formation process has identified several key parameters that characterize the droplet shape during the formation cycle. These include $L_{\text {lag }}^{*}$, the distance that the interface covers during the lag stage; $b_{\text {fill }}^{*}$, the penetration depth of the interface at the end of the filling stage; and $2 r_{\text {pinch }}^{*}$ and $b_{\text {pinch }}^{*}$, the critical thickness of the neck and the penetration depth at pinchoff, respectively. The variation of these parameters with geometry, fluid properties, and flow conditions are discussed next.

\section{Parameters influencing $L_{\text {lag }}^{*}$}

Observations show that the interface tends to pull back slightly into the side channel once a droplet detaches. This effect has not been previously reported most likely because the contribution to the final droplet volume is minimal $(\sim 0-5 \%)$. 
However, as the video analysis demonstrated, the lag period may have a significant contribution to the droplet spacing and must be included in the overall performance of the T-junction generator.

The geometry of the T-junction plays an important role in $L_{\text {lag }}^{*}$ purely due to the inherent shape of the back half of the droplet at detachment. Therefore, each generator design has its own unique dependence with $L_{\text {lag }}^{*}$. We noticed that for the same generator design $L_{\text {lag }}^{*}$ decreases with increasing dispersed phase flow $Q_{d} \uparrow$ or increasing dispersed phase viscosity $\mu_{d} \uparrow$. We hypothesize that this is caused by the competition between the pullback velocity of the interface right after rupture and the incoming dispersed phase flow.

Pinchoff in the T-junction generator shares many similarities with viscous-capillary pinchoff of two-fluid liquid bridges, which consist of a thin inner fluid thread of viscosity $\mu_{d}$ surrounded by an external fluid of viscosity $\mu_{c}$ [29,30]. The inner fluid drains from the thread at a rapid rate; however, it is opposed by the viscous drag created by the external fluid. Estimates suggests that the velocity within the thread scale as

$$
u_{\text {thread }} \sim \frac{\gamma \eta^{1 / 2}}{\mu_{d}} .
$$

In estimating the flow dependence of $L_{\text {lag }}^{*}$, we are interested in the pullback velocity of the tip right after rupture and not the draining flow within the thread. Postpinching flows are complex and not fully understood, but one can assume that the initial velocity of the receding interface will scale in a manner similar to Eq. (1) [31]. If we consider that the receding flow is opposed by the injection of the dispersed phase, it seems reasonable that $L_{\text {lag }}^{*}$ will depend on the relative strength of these two flows:

$$
L_{\mathrm{lag}}^{*} \sim \frac{u_{\mathrm{thread}}}{u_{\mathrm{DP}}}=\frac{\gamma \eta^{1 / 2}}{\mu_{d}} \frac{w_{d} h}{Q_{d}}=\frac{\eta^{1 / 2}}{\mathrm{Ca}_{d}},
$$

where $\mathrm{Ca}_{d}$ is the dispersed phase capillary number. Figure 4 plots the scaling relationship for a few T-junction geometries

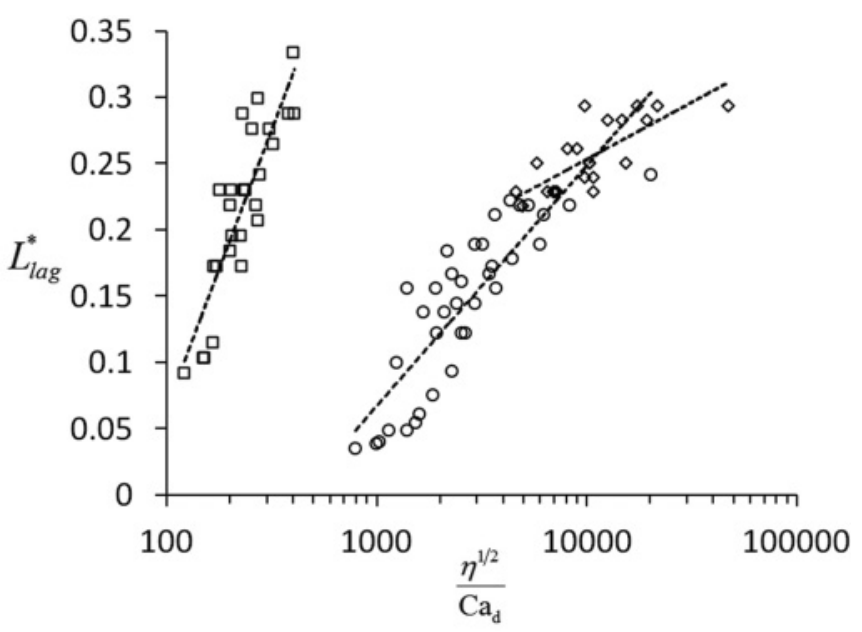

FIG. 4. Scaling relationship for $L_{\text {lag }}^{*}$ for three different T-junction geometries including the range of viscosity contrasts $\eta=0.12-1.7$. For geometries $\left(h^{*}, \Lambda^{*}\right)$, we have $(0.5,0.35)(\square),(0.4,1)(\circ)$, and $(0.3,1)(\diamond)$. over a range of flow conditions. For each design the data agrees with the proposed scaling analysis. However, more research is needed to understand the exact influence geometry and flow conditions on $L_{\text {lag }}^{*}$ in order to add its influence to a predictive model. This of course will require a more detailed study of the flow conditions during the final moments of collapse, which is beyond the scope of the current study.

\section{E. Parameters influencing $b_{\text {fill }}^{*}$}

Previous works have established that $b_{\text {fill }}^{*}$ may be determined by a force balance consisting of competing drag and surface tension forces acting on the interface. In their force balance,

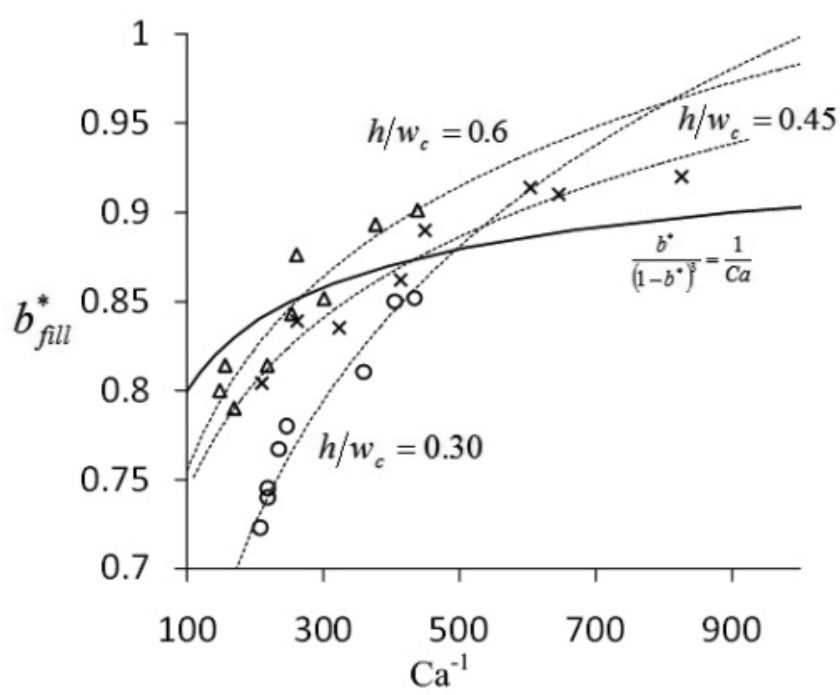

(a)

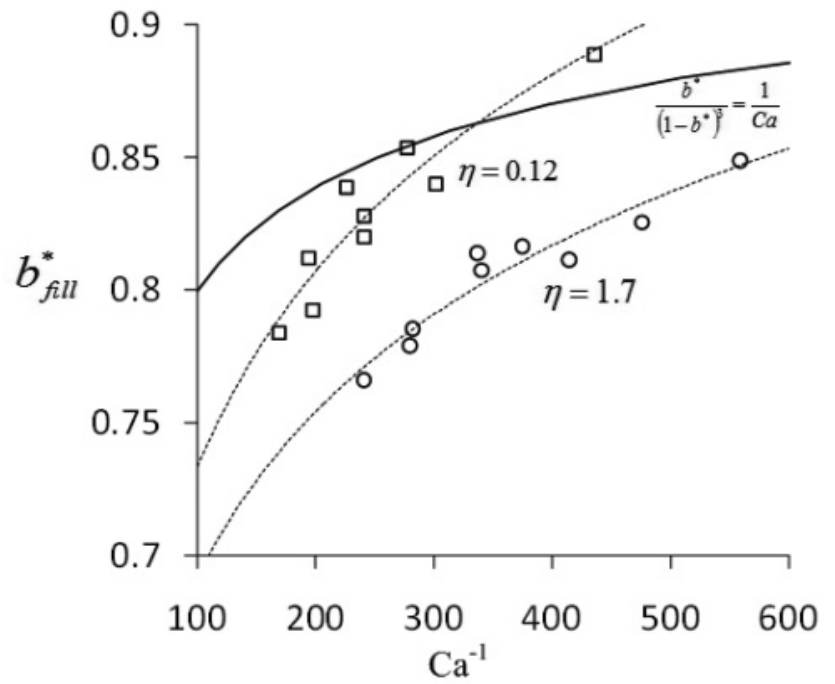

(b)

FIG. 5. Comparison between experimentally measured $b_{\text {fill }}^{*}$ and the expression by Christopher et al. [1]: (a) fluid combination of silicon oil and $10 \mathrm{wt} . \%$ glycerol-water mixture $(\eta=0.12)$ in different T-junction geometries demonstrating the dependence on $h / w_{c}$ and (b) effect of increasing the viscosity of the dispersed phase (10-70 wt.\% glycerol) for the same T-junction geometry $h / w_{c}=$ 0.45. Dashed lines are logarithmic curve fits used to guide the eye and the solid black line corresponds to the expression in Ref. [1]. 
Christopher et al. estimated that $b_{\text {fill }}^{*}$ scales with $1 / \mathrm{Ca}$ as [1]

$$
\frac{b_{\text {fill }}^{*}}{\left(1-b_{\text {fill }}^{*}\right)}=\frac{1}{\mathrm{Ca}} \text {. }
$$

However, when this function was compared to experimental data the fit was unsatisfactory, as shown in Fig. 5. First, the expression was unable to capture the influence of the aspect ratio $h / w_{c}$ on $b_{\text {fill }}$ [Fig. 5(a)] and second, the expression does not include the influence of the viscosity contrast [Fig. 5(b)]. Shallower channels and higher viscosity ratios result in a shift downward of $b_{\text {fill }}$ for the same flow conditions. Therefore, an alternative model of the formation process requires a more accurate representation of the force balance that includes these geometric and flow effects.

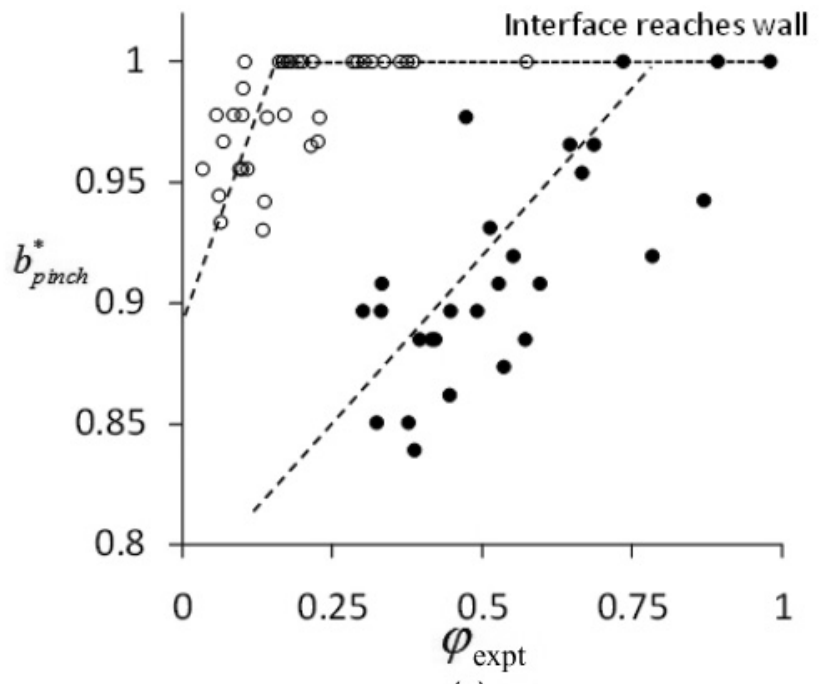

(a)

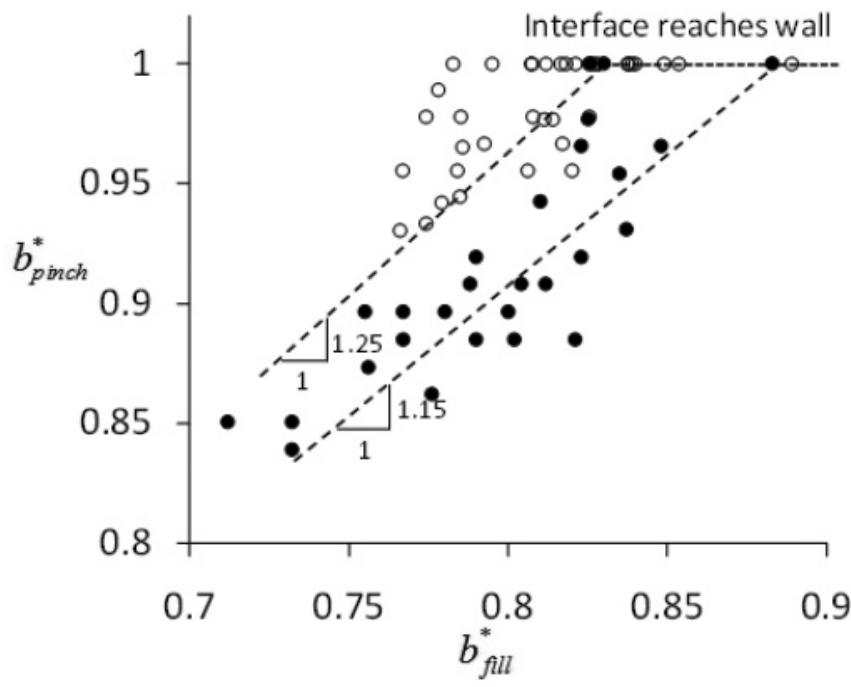

(b)

FIG. 6. Comparison between experimentally measured $b_{\text {pinch }}^{*}$ and (a) the flow ratio and (b) the initial penetration depth at the beginning of necking $b_{\text {fill }}^{*}$. For geometries $\left(h^{*}, \Lambda^{*}\right)$ we have $(0.30,0.35)(\bullet)$ and $(0.45,1)(\circ)$. Dashed lines are used to guide the eye to the linear relation.

\section{F. Parameters influencing $\boldsymbol{b}_{\text {pinch }}^{*}$}

During the necking process the interface continues to penetrate into the cross flowing stream causing $b^{*}$ to grow. The growth of $b^{*}$ is a result of the continuous rebalancing of forces as the curvature of the back half of the droplet changes combined with the increasing volume of the droplet. Furthermore, the time that $b^{*}$ has to grow will also depend on the duration of the necking stage, which scales with the flow ratio $V_{\text {drop }} \sim Q_{d} / Q_{c}$, and therefore one expects to see such a dependence on $b_{\text {pinch. }}^{*}$. Figure 6(a) plots the relationship between $b_{\text {pinch }}^{*}$ and $\varphi$ for two different geometries, one with a short necking time $\left(\Lambda^{*}=0.3\right)$ and the other with a long necking time $\left(\Lambda^{*}=1\right)$. The latter case results in larger values of $b_{\text {pinch }}^{*}$ for the same flow ratio, as expected.

The issue with trying to develop a model to predict $b_{\text {pinch }}^{*}$ is that a reciprocal relationship exists between the necking time, force balance, flow rate, which would require some sort of iteration to solve. However, if we plot $b_{\text {pinch versus the }}^{*}$ initial size of the droplet at the beginning of necking $b_{\text {fill }}^{*}$, we see that there is almost a linear relationship between the two parameters. For the two geometries $\left(\Lambda^{*}=0.35\right.$ and 1$)$, $b_{\text {pinch }}^{*}$ increases by approximately $15-25 \%$ over $b_{\text {fill }}^{*}$. This simple correlation is used later in the model to avoid the unwanted complexity associated with iteration.

\section{G. Parameters influencing $2 r_{\text {pinch }}^{*}$}

Experiments performed by van Steijn et al. demonstrated that the neck begins to pinch off once the difference in Laplace pressure from the tip to the neck of the droplet causes the bypassing continuous phase to reverse direction [12]. The additional flow into the neck region accelerates the rate of collapse and pinchoff occurs almost immediately afterward. The authors estimated the critical moment of the flow reversal by determining when the Laplace pressure difference between

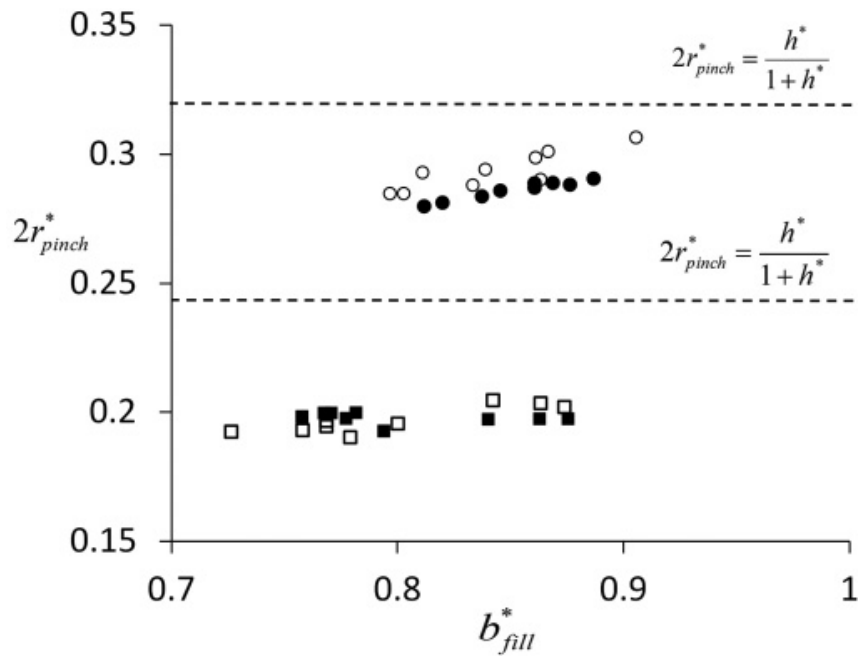

FIG. 7. Comparison between experimental measured $2 r_{\text {pinch }}^{*}$ and penetration depth at the beginning of necking $b_{\text {fill }}^{*}$. For geometries $\left(h^{*}, \Lambda^{*}\right)$ we have $(0.4,1)(\circ)$ and $(0.30,0.35)(\square)$. Open symbols correspond to $\eta=0.12$ and closed symbols correspond to $\eta=1.7$. Dashed lines are the theoretical limit predicted by Eq. (4). 
the tip and neck equals zero. This coincides with the neck reaching a critical shape

$$
2 r_{\text {pinch }}^{*}=\frac{h^{*}}{1+h^{*}} \text {. }
$$

The expression suggests that pinchoff is determined only by the geometry of the generator. However, when Eq. (4) was applied in the present study, it consistently overpredicted the critical thickness that was observed as shown in Fig. 7. Data are plotted against $b_{\text {fill }}^{*}$, which was chosen because it includes the combined influence of flow conditions $(\mathrm{Ca}, \eta)$ and the shape of the droplet near pinchoff. The data suggest that there is a relationship between the flow conditions and $2 r_{\text {pinch }}^{*}$ that is not included in Eq. (4).

We also note that $2 r_{\text {pinch }}^{*}$ displays an insensitivity to the dispersed phase viscosity, which confirms the proposal that pinchoff is primarily dictated by the flow of the continuous phase around the drop and not the internal flow of the dispersed phase. The viscosity of the dispersed phase is expected to come into play during the final pinch-off moments where the rate of

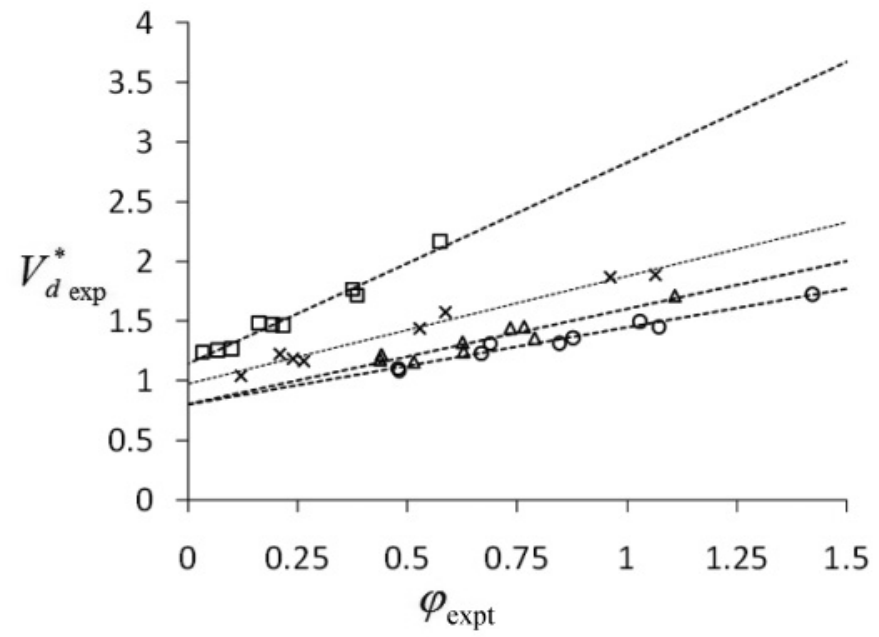

(a)

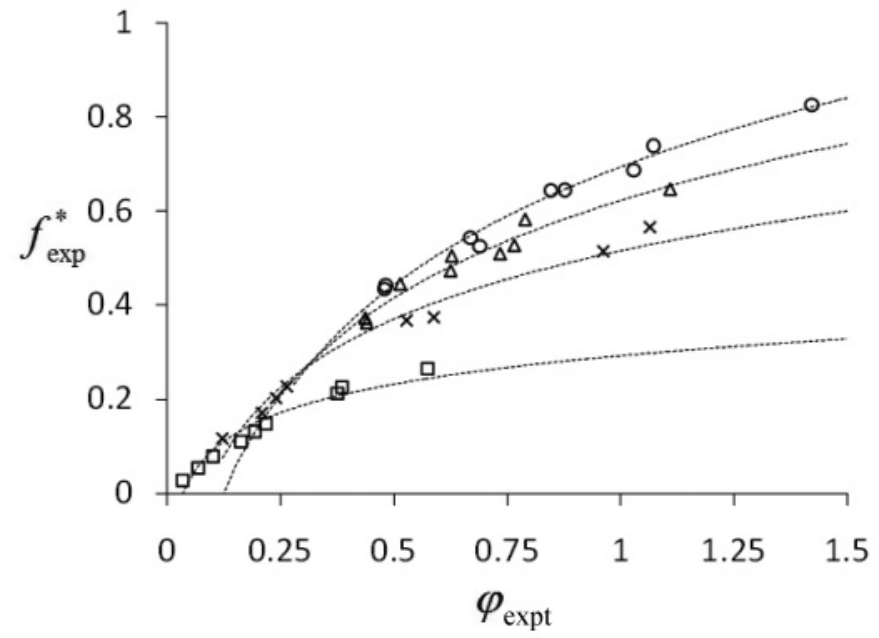

(c) thinning of the neck resembles the conditions present in the two-fluid liquid bridge. However, for the purpose of predicting the operation of the T-junction generator, the final moments leading to rupture of the neck are not important because of the relatively short time scale compared to the rest of the formation process. The most important matter is to precisely calculate the moment when the pinch-off process begins, which requires an alternative model for $2 r_{\text {pinch }}^{*}$.

\section{H. Scaling of operational parameters}

The scaling of operational parameters for the four generator designs is presented in Fig. 8. Each generator follows the general scaling law $V_{d}^{*}=\alpha+\beta \phi$, but with different fitting parameters defining the slope and intercept depending primarily on the T-junction design. Dashed lines in the figure are curve fits to the data. Scatter around these base lines is caused by experimental variance as well as $\mathrm{Ca}$ dependence, which is not included in the plots.

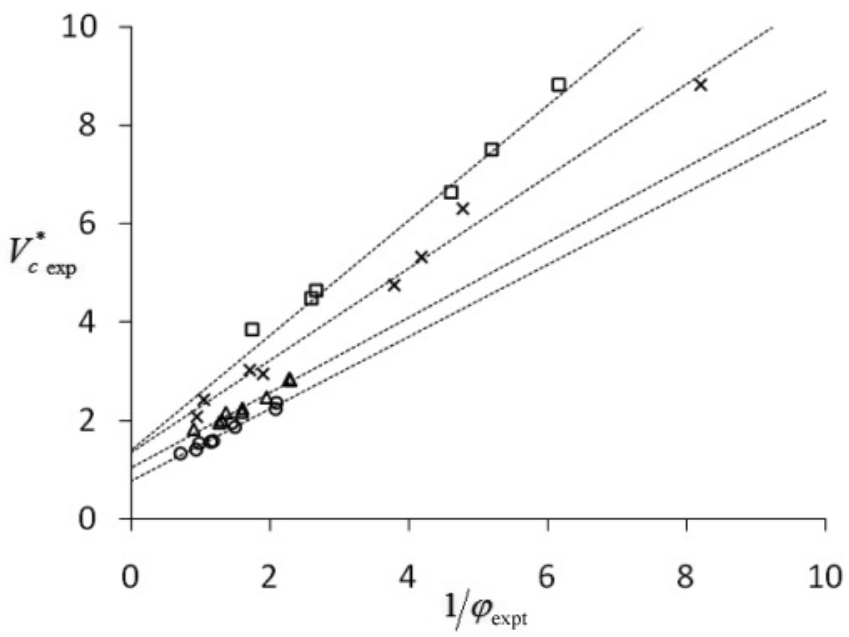

(b)

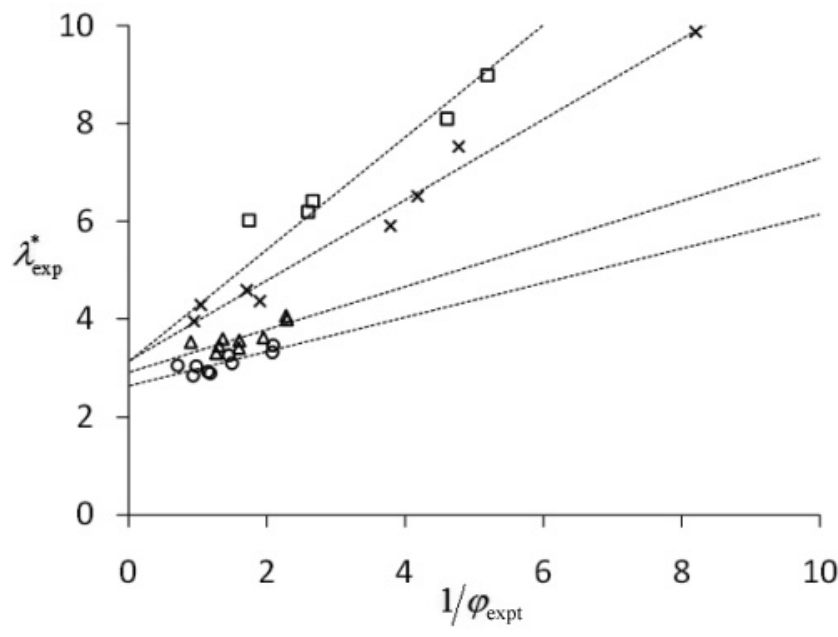

(d)

FIG. 8. Plot of the (a) dimensionless droplet volume, (b) oil volume, (c) frequency of formation, and (d) spacing as a function of the flow ratio. For geometries $\left(h^{*}, \Lambda^{*}\right)$ we have $(0.4,1)(\square),(0.30,0.35)(\circ),(0.6,0.5)(\Delta)$, and $(0.5,0.5)(\times)$, with silicone oil and 10 wt.\% glycerol-water mixture as the working fluids. Dashed lines are fits to guide the eye. 
According to analysis of the formation process, the continuous phase volume and droplet spacing are expected to vary proportionally with the $Q_{c}$ and inversely with $Q_{d}$. The plots show that both the oil volume and droplet spacing scale linearly with the inverse of the flow ratio $\varphi$, as expected. The intercept and slope are exchanged from the droplet volume scaling $V_{c}^{*}=\beta+\alpha / \phi$.

The frequency is plotted as a dimensionless number scaled by the characteristic residence time in the channel $t^{-1}=$ $Q_{c} / w_{c}^{2} h$. Since the formation process is governed by the flow of both phases, it is reasonable to expect that the frequency will also scale with the flow ratio. As shown in Fig. 8(c), the frequency does in fact scale with $\varphi$, but in a nonlinear manner trending to an asymptote at high flow ratios.

\section{CONCLUSION}

In this first of two papers we have presented a detailed analysis of the operation of microfluidic T-junction generators operating in the transitional regime. The drop formation process was divided into three stages: the lag stage, where the interface recovers after detachment; the filling stage, where the interface penetrates into the main channel; and the necking stage, which eventually leads to pinchoff. Our analysis identified several key parameters that describe the droplet shape during the formation process and we have analyzed the dependence on geometric and flow conditions. In the following paper we will present a model based on our observations that explains the complete operation of microfluidic T-junction generators in the transition regime [26].
[1] G. F. Christopher, N. N. Noharuddin, J. A. Taylor, and S. L. Anna, Phys. Rev. E 78, 036317 (2008).

[2] S. Y. Teh, R. Lin, L. H. Hung, and A. P. Lee, Lab. Chip. 8, 198 (2008).

[3] L. Shui, J. C. T. Eijkel, and A. van den Berg, Adv. Colloid. Interface Sci. 133, 35 (2007).

[4] S. Haeberle and R. Zengerle, Lab. Chip. 7, 1094 (2007).

[5] G. F. Christopher and S. L. Anna, J. Phys. D 40, R319 (2007).

[6] M. De Menech, P. Garstecki, F. Jousse, and H. A. Stone, J. Fluid Mech. 595, 141 (2008).

[7] P. Garstecki, M. J. Fuerstman, H. A. Stone, and G. M. Whitesides, Lab. Chip. 6, 437 (2006).

[8] J. H. Xu, S. W. Li, J. Tan, and G. S. Luo, Microfluid. Nanofluid. 5, 711 (2008).

[9] M. Zagnoni, J. Anderson, and J. M. Cooper, Langmuir 26, 9416 (2010).

[10] V. van Steijn, M. T. Kreutzer, and C. R. Kleijn, Chem. Eng. Sci. 62, 7505 (2007).

[11] T. T. Fu, Y. G. Ma, D. Funfschilling, C. Y. Zhu, and H. Z. Li, Chem. Eng. Sci. 65, 3739 (2010).

[12] V. van Steijn, C. R. Kleijn, and M. T. Kreutzer, Phys. Rev. Lett. 103, 214501 (2009).

[13] V. van Steijn, C. R. Kleijn, and M. T. Kreutzer, Lab. Chip. 10, 2513 (2010).

[14] A. Gupta and R. Kumar, Microfluid. Nanofluid. 8, 799 (2010).

[15] M. L. J. Steegmans, C. Schroen, and R. M. Boom, Chem. Eng. Sci. 64, 3042 (2009).

[16] D. Fries and P. R. von Rohr, Microfluid. Nanofluid. 6, 27 (2009).
[17] S. van der Graaf, T. Nisisako, C. Schroen, R. G. M. van der Sman, and R. M. Boom, Langmuir 22, 4144 (2006).

[18] K. Wang, Y. C. Lu, J. H. Xu, and G. S. Luo, Langmuir 25, 2153 (2009).

[19] Y. X. Zhang and L. Q. Wang, Nano Micro. Thermophys. Eng. 13, 228 (2009).

[20] L. Dai, W. F. Cai, and F. Xin, Chem. Eng. Technol. 32, 1984 (2009).

[21] J. Husny and J. J. Cooper-White, J. Non-Newtonian Fluid Mech. 137, 121 (2006).

[22] L. Sang, Y. P. Hong, and F. J. Wang, Microfluid. Nanofluid. 6, 621 (2009).

[23] M. L. J. Steegmans, K. Schroen, and R. M. Boom, Langmuir 25, 3396 (2009).

[24] A. Gupta, S. M. S. Murshed, and R. Kumar, Appl. Phys. Lett. 94, 164107 (2009).

[25] T. Schneider, D. R. Burnham, J. VanOrden, and D. T. Chiu, Lab. Chip. 11, 2055 (2011)

[26] T. Glawdel, C. Elbuken, and C. Ren, Phys. Rev. E 85, 016323 (2012).

[27] R. Dangla, F. Gallaire, and C. N. Baroud, Lab. Chip. 10, 2972 (2010).

[28] See Supplemental Material at http://link.aps.org/supplemental/ 10.1103/PhysRevE.85.016322 for experimental details regarding channel height measurements and video analysis.

[29] A. Sierou and J. R. Lister, J. Fluid Mech. 497, 381 (2003).

[30] J. R. Lister and H. A. Stone, Phys. Fluids 10, 2758 (1998).

[31] D. T. Papageorgiou, J. Fluid Mech. 301, 109 (1995). 